\title{
Alterações em variáveis cardiovasculares e intracranianas induzidas pela associação de buprenorfina e sevoflurano em cães
}

\section{Alterations on cardiovascular and intracranial variables caused by buprenorphine and sevoflurane association in dogs}

\author{
Newton Nunes," Almir Pereira de Souza," Márlis Langenegger de Rezende," Paulo Sérgio Patto dos Santos,"
} Danielli Parrilha de Paula," Celina Tie Nishimori"

\begin{abstract}
Resumo
Objetivou-se, com este experimento, estudar possiveis alterâçôes nas variáveis cardiovasculares e intracranianas promovidas pela buprenorfina, em cães anestesiados com sevoflurano. Utilizaram-se oito cães adultos, clinicamente saudáveis. A anestesia foi induzida com propofol ( $8 \mathrm{mg} / \mathrm{kg}$ IV) e em seguida os animais foram entubados com sonda orotraqueal de Magill, a qual foi conectada ao aparelho de anestesia volátil para administração de sevoflurano (1,3 CAM). Os animais foram mantidos sob ventilação controlada durante o período experimental. Após 20 minutos do posicionamento do cateter de pressão intracraniana (PIC), foi aplicada buprenorfina $(0,02 \mathrm{mg} / \mathrm{kg}$ IV). Avaliou-se: PIC; temperatura intracraniana (TIC); pressão de perfusão cerebral (PPC); freqüência cardíaca $(F C)$; pressões arteriais sistólica, média e diastólica (PAS, PAM e PAD, respectivamente); débito cardiaco (DC); pressão venosa central (PVC); pressão da artéria pulmonar (PAP); e temperatura corporal (TC). As colheitas foram feitas nos seguintes momentos: M1 - 20 minutos após ó posicionamento do cateter de PIC; M2 - 15 minutos após a administração do opióide; M3, M4 e M5 - de 15 em 15 minutos após M2. A avaliação estatística dos dados foi efetuada por meio do Teste de Tukey $(P<0,05)$. A PIC permaneceu estável durante todo o período experimental, sendo observada discreta elevação em M2, mas sem significado clínico ou estatístico. Entretanto, registrou-se uma queda acentuada, estatisticamente significativa, da PPC após o M2. As variáveis cardiovasculares FC, PAS, PAM, PAD, DC e PAP apresentaram redução significativa de seus valores após $M 2$, mantendo-se estáveis no restante do periodo experimental. Quanto à PVC, o teste estatístico não registrou alterações significativas, porém pode-se observar uma discreta elevação desta variável após a aplicação da buprenorfina. Assim, pode-se concluir que a buprenorfina não interferiu na PIC. Entretanto, a queda da PAM, determinada pela administração do opióide, causa redução da PPC em cães anestesiados com sevoflurano. As demais variáveis cardiovasculares não sofreram influência do opióide.
\end{abstract}

Palavras-chave: buprenorfina, sevoflurano, cães, anestesia.

\begin{abstract}
The aim of this work was to study the possible alterations on cardiovascular and intracranial variables caused by buprenorphine, in dogs anesthetized with sevoflurane. Eight healthy adult mongrel dogs were used. Anesthesia was induced with propofol $(8 \mathrm{mg} / \mathrm{kg} / \mathrm{lV})$, and maintained with sevoflurane (1.3 MAC). The animals were mechanically ventilated during all the experimental period. The dogs were intrumented and 20 minutes after the introduction of the intracranial catheter, they received buprenorphine $(0.02 \mathrm{mg} / \mathrm{kg} / \mathrm{IV})$. Intracranial pressure (ICP), intracranial temperature (ICT), cerebral perfusion pressure (CPP), heart rate (HR), systolic, diastolic and mean arterial pressure (SAP, DAP and MAP), cardiac output (CO), central venous pressure (CVP), pulmonary artery pressure (PAP) and body temperature (BT) were evaluated. The measurements were realized 20 minutes after the introduction of the intracranial catheter (M1), 15 minutes after buprenorphine administration (M2) and every 15 minutes after M2 (M3, M4, M5). The numerical data were submitted to Tukey Test $(p<0.05)$. Intracranial pressure was stable during all the experimental period, being observed only a slight increase after buprenorphine administration (M2). However, this increase was without any statistical meaning. In the other hand, a marked decrease of cerebral perfusion pressure after M2 was recorded. The cardiovascular variables HR, SAP, DAP, MAP, $C O$ and PAP presented a significative reduction after M2 and were stable during the rest of the experimental period. Regarding CVP, no statistical differences were recorded, but it was possible to notice a discreet increase after buprenorphine administration. The results allow us to conclude that buprenorphine didn't interfere with intracranial pressure. Although, the marked decrease of arterial pressure due to buprenorphine administration caused a reduction of the cerebral perfusion pressure in dogs anesthetized with sevoflurane. The other variables didn't suffer any influence of buprenorphine.
\end{abstract}

Keywords: buprenorphine, sevoflurane, dogs, anesthesia.

\footnotetext{
* Departamento de Clínica e Cirurgia Veterinária, Faculdade de Ciências Agrárias e Veterinárias - UNESP - Campus de Jaboticabal - São Paulo. newton@fcav.unesp.br (autor para correspondência)

** Pós-graduando(a) em Cirurgia Veterinária, Departamento de Clínica e Cirurgia Veterinária, Faculdade de Ciências Agrárias e Veterinárias UNESP - Campus de Jaboticabal - São Paulo.
} 


\section{Introdução}

Diversos agentes analgésicos opióides têm sido colocados à disposição da classe médica e veterinária. Desta forma, abrem-se possibilidades variadas do uso isolado ou associado destes fármacos aos agentes anestésicos inalatórios da atualidade.

A buprenorfina é um opióide com atividade agonista e antagonista no homem e nos animais (Mar'inez et al., 1997), com potência analgésica aproximadamente 30 vezes maior que a da morfina (Thurmon et al., 1996), aliviando a dor de moderada a grave, associada a procedimentos cirúrgicos abdominais, torácicos e ortopédicos (Górniak, 1997). Um dos efeitos hemodinâmicos conseqüentes da administração deste opióide, em cães, é a diminuição significativa da freqüência cardiaca, provavelmente por aumento do tono vagal, e alterações discretas na pressão sangüínea sistêmica (Stepien et al.,1995). A depressão respiratória, passível de ocorrer em algumas situações, pode ser revertida com o uso de antagonistas opióides, como o naloxone (Thurmon et al., 1996).

O sevoflurano é um éter isopropílico fluoretado não-inflamável, de peso molecular 200 , ponto de ebulição aos $58,5^{\circ} \mathrm{C}$ e pressão de vapor de aproximadamente $162 \mathrm{mmHg}$ a $20^{\circ} \mathrm{C}$. Sobre o sistema cardiovascular, o fármaco promove queda da pressão arterial média, deprime a contractilidade do miocárdio e não sensibiliza o miocárdio à ação das catecolaminas (Navarro et al., 1994), enquanto que Kawana et al. (1995) e Bernard et al. (1990) detectaram ligeiro aumento na freqüência cardíaca. Comparado aos demais anestésicos voláteis, parece ser o que oferece maior estabilidade cardiovascular (Ebert et al., 1995).

A depressão respiratória produzida pelo agente anestésico ocorre devido à sua ação direta sobre o centro ventilatório medular e indireta sobre os músculos intercostais (Omoigui, 1998). A vasodilatação cerebral promovida pelo sevoflurano causa aumento no fluxo e volume sangüineos cerebrais, levando ao aumento da pressão intracraniana. Com o tempo, a autorregulação vascular cerebral suaviza o aumento de fluxo sangüineo cerebral, à semelhança do que ocorre com o isoflurano (Omoigui, 1998).

Baseando-se nas novas informações relativas aos fármacos em pauta, objetivou-se, com a realização deste estudo, avaliar as possiveis interferências na dinâmica cardiopulmonar e nas variáveis intracranianas determinadas pela administração do opióide buprenorfina, em cães, durante anestesia com desflurano.

\section{Material e métodos}

Foram utilizados oito cães adultos, machos e fêmeas, clinicamente saudáveis, com peso médio de $11,5 \mathrm{~kg}( \pm 2,7)$. Após jejum alimentar de 12 horas e restrição hídrica de duas horas, estes foram induzidos com $8 \mathrm{mg} / \mathrm{kg}$ de propofol, ${ }^{1}$ por via intravenosa (IV), e entubados com sonda de Magill. Ato contínuo, cateterizou-se a veia cefálica e aplicou-se rocurônio ${ }^{2}$ na dose de $0,6 \mathrm{mg} / \mathrm{kg}$ IV. A sonda orotraqueal foi acoplada à aparelho de anestesia inalatória, ${ }^{3}$ equipado com ventilador

\footnotetext{
1 DIPRIVAN - ZENECA Farmacêutica do Brasil Ltda.

2 ESMERON - ORGANON TEKNIKA B.V.IAKZO NOBEL Ltda.
}

volumétrico ${ }^{4}$ e iniciou-se a administração do sevoflurano ${ }^{5}$ (1,3 CAM) por meio de vaporizador calibrado. ${ }^{6}$ Procedeu-se a ventilação controlada, com amplitude e freqüência suficientes para permitir leitura de capnometria constante em $35 \mathrm{mmHg}$, aferida em oxicapnógrafo ${ }^{7}$ cujo sensor de fluxo principal foi posicionado na extremidade da sonda orotraqueal conectada ao equipamento de anestesia. O bloqueio neuromuscular foi misntido durante todo o periodo experimental através de infusão contínua de rocurônio $(0,6 \mathrm{mg} / \mathrm{kg} / \mathrm{h})$ sendo, para tanto, utilizada bomba de infusão contínua. $O$ controle da concentração anestésica foi obtido por meio de analisador de gases, ${ }^{8}$ cujo sensor foi colocado em série com o sensor de capnometria. Foi realizada a monitorização dos animais e 20 minutos após o posicionamento do cateter de pressão intracraniana, foi administrada a buprenorfina ${ }^{9}(0,02$ $\mathrm{mg} / \mathrm{kg} / \mathrm{lV}$ ). Durante toda a fase experimental a temperatura foi mantida estável através de colchão térmico. ${ }^{10} \mathrm{O}$ protocolo experimental foi baseado naquele estabelecido por Gross et al. (1993) e Greene et al. (1990).

Foram avaliados: pressão intracraniana (PIC), através de mensuração digital direía em monitor de pressão intracraniana, ${ }^{11}$ cujo cateter de fibra óptica ${ }^{12}$ foi implantado cirurgicamente, com o uso de kit de acesso, ${ }^{13}$ na superfície do córtex cerebral direito, através de técnica descrita por Bagley et al. (1995); temperatura intracraniana (TIC) obtida pelo monitor empregado para a $\mathrm{RIC}$; pressão de perfusão craniana (PPC), obtida pela diferença entre a pressão arterial média e PIC; freqüência cridíaca (FC), obtida em monitor de eletrocardiografia computadorizado, ${ }^{14}$ pelo cálculo do intervalo R-R; pressões arteriais sistólica, média e diastólica (PAS, $P A M$ e PAD, respectivamente), obtida pela cateterização da artéria femoral esquerda ou direita, cuja leitura foi feita em monitor digital computadorizado, ${ }^{15}$ débito cardíaco (DC), obtida após introdução do cateter de Swan-Ganz pela veia femoral (esquerda ou direita) até o correto posicionamento na artéria pulmonar, cuja leitura, foi obtida através do mesmo monitor utilizado para pressão arterial; pressão venosa central (PVC) e pressão da artéria pulmonar (PAP), ambas obtidas pelo mesmo cateter utilizado para o DC; e temperatura corporal (TC), obtida pelo sensor de temperatura localizada na extremidade do cateter de Swan- $\mathcal{G}_{i}$ anz, adotado para a mensuração do débito cardíaco.

As determinações foram feitas nos seguintes momentos: M1 - 20 minutos após o implante do cateter de PIC, imediatamente antes da administração do fármaco opióide; $M 2-15$

\footnotetext{
${ }^{3}$ OHMEDA - mod. Excel 210SE - Processo FAPESP 97/10668 - 4

4 OHMEDA - mod. 7900 - processo FAPESP 97/10668 - 4

${ }^{5}$ SEVORANE - ABBOTT Laboratórios do Brasil Ltda.

6 OHMEDA - mod. Sevotec 5

7 DIXTAL - mod. CO $\mathrm{CO}_{2}$ SMO 7100 - Processo FAPESP 97/10668-4

8 OHMEDA - mod. 5250 RGM - Processo FAPESP 96/12830-0

9 TEMGESIC - Ind. Quim. e Farm. SCHERING-PLOUGH SIA

10 GAYMAR - Model TP5nO

$"$ CAMINO LABS - mod. MPM - 1 - Proc. FAPESP 00/01084-3

12 CAMINO LABS - mod. 110 - 4BT - Proc. FAPESP 00/01084-3

${ }^{13}$ CAMINO LABS - mod. 5H-ITH - Proc. FAPESP 00/01084-3

14 TEB - Model ECGPC 1.10 - Proc. FAPESP 97/10668-4

15 DIXTAL DX 2010 - Proc. FAPESP 97/10668-4
} 
minutos após a administração do opióide; M3, M4 e M5 - de 15 em 15 minutos após M2. A avaliação estatística dos dados foi efetuada por meio de Análise de Variância (ANOVA), seguida do Teste de Tukey $(P<0,05)$.

\section{Resultados}

A PIC (Tabela 1) permaneceu estável durante todo o período experimental. Foi observada uma discreta elevação da pressão (de $18 \mathrm{mmHg} \pm 3,93 \mathrm{em} \mathrm{M1}$ para 19,1 $\mathrm{mmHg} \pm 7,16 \mathrm{em}$ M2) após a administração do opióide, não diferindo, entretanto, pela análise estatística empregada. Registrou-se uma redução estatisticamente significativa da PPC após o M2 $(57,1$ $\mathrm{mmHg} \pm 21,92)$, que se manteve ao longo de todo o período experimental. Não foram observadas alterações dignas de nota da TIC (Tabela 1). A temperatura em M1, que era de 36,9 $( \pm 0,91)$, apresentou uma redução imperceptível após a aplicação do opióide $(36,80 \pm 0,88)$, permanecendo neste patamar durante os demais momentos.

Tabela 1 - Valores médios e desvios-padrão (entre parênteses) da pressão intracraniana (PIC), pressão de perfusão cerebral (PPC) e temperatura intracraniana (TIC), de cães medicados com buprenorfina durante anestesia com sevoflurano, sob ventilação controlada.

\begin{tabular}{cccccc}
\hline Variável & M1 & M2 & M3 & M4 & M5 \\
\hline PIC & $18,0 \mathrm{a}$ & $19,1 \mathrm{a}$ & $19,5 \mathrm{a}$ & $19,2 \mathrm{a}$ & $19,4 \mathrm{a}$ \\
$(\mathrm{mmHg})$ & $(3,93)$ & $(7,16)$ & $(7,39)$ & $(7,05)$ & $(6,55)$ \\
\hline $\mathrm{PPC}(\mathrm{mmHg})$ & $75,5 \mathrm{a}$ & $57,1 \mathrm{~b}$ & $54,9 \mathrm{~b}$ & $58,1 \mathrm{~b}$ & $58,0 \mathrm{~b}$ \\
& $(20,72)$ & $(21,92)$ & $(20,46)$ & $(20,25)$ & $(21,80)$ \\
\hline $\mathrm{TIC}$ & $36,9 \mathrm{a}$ & $36,8 \mathrm{a}$ & $36,7 \mathrm{a}$ & $36,6 \mathrm{a}$ & $36,6 \mathrm{a}$ \\
$\left({ }^{\circ} \mathrm{C}\right)$ & $(0,91)$ & $(0,88)$ & $(0,87)$ & $(0,81)$ & $(0,77)$ \\
\hline
\end{tabular}

Para cada variável, médias seguidas pela mesma letra, não diferem significativamente pelo teste de Tukey $(P<0,05)$.

No tocante à FC (Tabela 2), pode-se observar um acentuado decréscimo de seus valores após M2 $(91,9 \pm 14,02)$, quando comparado a seus índices iniciais $(M 1=112,1 \pm 18,31)$. Houve uma estabilidade dos dados após a queda registrada inicialmente (M2 a M5). Mesmo comportamento pode ser observado quando se analisam os dados referentes à pressão arterial (Tabela 2), a partir de $M 2$ (PAS $=112,2 \pm 20,30 ; P A D=60,9$ $\pm 17,46$; PAM $=80,0 \pm 16,56$; e PAP $=12,9 \pm 3,23)$. A PAD continuou a manifestar tendências a queda, apesar de o teste estatístico não ter detectado diferenças ao longo dos momentos, após M2. O DC foi outra variável cardiovascular que seguiu a mesma tendência de redução registrada nas variáveis citadas anteriormente, a partir da aplicação da buprenorfina ( $M 2=1,7 \pm 0,42$ ). Houve, de modo semelhante ao ocorrido com as variáveis anteriores, uma estabilização dos valores, não apresentando nenhuma alteração estatisticamente significativa. Com relação à variável PVC pode-se constatar um fenômeno inverso daquele registrado nos outros parâmetros, ou seja, a partir de M2 ocorreu uma discreta elevação de seus valores ( $M 2=3,9 \pm 1,81$ ), quando comparado ao momento inicial $(M 1=3,252 \pm 1,67)$. Entretanto, estes achados não foram estatisticamente diferentes entre si.

A temperatura corporal iniciou-se com $36,8^{\circ} \mathrm{C}( \pm 0,97)$, permanecendo neste patamar nos momentos seguintes, até findar em $36,5^{\circ} \mathrm{C}( \pm 0 ; 76)$ em M5.
Tabela 2 - Valores médios e desvios-padrão (entre parênteses) da frequeência cardíaca ( $F C$ ), pressão arterial sistólica, média e diastólica (PAS, PAM e PAD), débito cardíaco (DC), pressão venosa central (PVC), pressão da artéria pulmonar (PAP) e temperatura corporal (TC), de cães medicados com buprenorfina durante anestesia com sevoflurano, sob ventilação controlada.

\begin{tabular}{cccccc}
\hline Variável & M1 & M2 & M3 & M4 & M5 \\
\hline FC (bat./min.) & $112,1 \mathrm{a}$ & $91,9 \mathrm{~b}$ & $89,7 \mathrm{~b}$ & $91,5 \mathrm{~b}$ & $91,1 \mathrm{~b}$ \\
& $(18,31)$ & $(14,02)$ & $(11,94)$ & $(14,53)$ & $(12,83)$ \\
PAS (mmHg) & $127,7 \mathrm{a}$ & $112,2 \mathrm{~b}$ & $110,1 \mathrm{~b}$ & $111,6 \mathrm{~b}$ & $110,6 \mathrm{~b}$ \\
& $(22,02)$ & $(20,30)$ & $(17,54)$ & $(15,55)$ & $(18,33)$ \\
PAD (mmHg) & $72,1 \mathrm{a}$ & $60,9 \mathrm{~b}$ & $57,6 \mathrm{~b}$ & $59,0 \mathrm{~b}$ & $58,2 \mathrm{~b}$ \\
& $(16,89)$ & $(17,46)$ & $(15,15)$ & $(15,54)$ & $(16,67)$ \\
PAM (mmHg) & $93,5 \mathrm{a}$ & $80,0 \mathrm{~b}$ & $77,9 \mathrm{~b}$ & $79,2 \mathrm{~b}$ & $79,1 \mathrm{~b}$ \\
& $(17,78)$ & $(16,56)$ & $(15,81)$ & $(15,31)$ & $(17,45)$ \\
DC & $2,2 \mathrm{a}$ & $1,7 \mathrm{~b}$ & $1,6 \mathrm{~b}$ & $1,7 \mathrm{~b}$ & $1,7 \mathrm{~b}$ \\
$(\mathrm{~L} / \mathrm{min})$. & $(0,55)$ & $(0,42)$ & $(0,26)$ & $(0,28)$ & $(0,41)$ \\
PVC (mmHg) & $3,2 \mathrm{a}$ & $3,9 \mathrm{a}$ & $3,6 \mathrm{a}$ & $4,2 \mathrm{a}$ & $3,5 \mathrm{a}$ \\
& $(1,67)$ & $(1,81)$ & $(2,07)$ & $(1,49)$ & $(2,07)$ \\
PAP (mmHg) & $15,4 \mathrm{a}$ & $12,9 \mathrm{~b}$ & $12,9 \mathrm{~b}$ & $13,0 \mathrm{~b}$ & $13,0 \mathrm{~b}$ \\
& $(4,03)$ & $(3,23)$ & $(2,95)$ & $(2,78)$ & $(3,12)$ \\
TC & $36,8 \mathrm{a}$ & $36,7 \mathrm{a}$ & $36,6 \mathrm{a}$ & $36,5 \mathrm{a}$ & $36,5 \mathrm{a}$ \\
( $\mathrm{C})$ & $(0,97)$ & $(0,96)$ & $(0,87)$ & $(0,80)$ & $(0,76)$ \\
\hline
\end{tabular}

Para cada variável, médias seguidas pela mesma letra na linha, não diferem significativamente pelo teste de Tukey $(P<0,05)$.

\section{Discussão}

Embora não se tenha utilizado um grupo de animais recebendo sevoflurano, acrescido de placebo, de modo a simular a administração de buprenorfina, a metodologia pôde ser considerada adequada, para que se atingissem os objetivos, uma vez que se protocolou o método conforme sugerido por Gross et al. (1993) e Greene et al. (1990). Os autores basearam as mensurações em teste comparativo entre vários momentos e aquele inicial, quando os pacientes haviam recebido apenas $O$ anestésico volátil. A repetição deste método forneceu valores considerados confiáveis e permitiu o cotejamento das variáveis entre os momentos, permitindo reduzir o número de animais, o que é desejável, do ponto de vista ético e minimizando os custos do experimento

Os achados referentes à $\mathrm{PIC}$, embora não dotados de diferença estatística significativa, merecem melhor detalhamento. Neste sentido, o aumento discreto, observado em M2 pode ser imputado à buprenorfina. Parece evidente que o opióide não é dotado de potencial nocivo, quanto ao aumento da PIC, uma vez que os valores encontrados a partir de sua administração encoliram-se próximos o suficiente dos considerados fisiológicos, para a espécie. Ainda relativamente à PIC, cabe lembrar que com o protocolo proposto estabeleceu-se o momento inicial com os cães já anestesiados pelo sevoflurano. Tal situação permitiu identificar valores iniciais de PIC ao redor de $18 \mathrm{mmHg}$; entretanto, é conhecido o efeito do anestésico sobre a vasodilatação cerebral (Omoigui, 1998), o que poderia ter determinado aumento, embora discreto, da pressão intracraniana em $\mathrm{M} 1$, impedindo o aparecimento de diferenças estatísticas significativas, na comparação com os demais momentos, onde os efeitos da buprenorfina já se fariam presentes.

Optou-se por realizar o experimento em condições de normocapnia, com o emprego de ventilação controlada, me- 
diante 0 uso de bloqueio neuromuscular periférico. $O$ miorrelaxante empregado não é dotado de características que possam interferir sobre as variáveis intracranianas (PIC e PPC) e pressão arterial média (Schramm et al., 1996). Sendo assim, é possivel especular quanto a possiveis interferências da buprenorfina sobre a ventilação espontânea, o que poderia levar ao aumento dos níveis arteriais de dióxido de carbono, com conseqüente elevação da PIC, caso o opióide determinasse redução na frequêencia ou amplitude da respiração, como descrito por Thurmon et al. (1996). Desta forma, os efeitos da associação entre o sevoflurano e a buprenorfina, em pacientes sob ventilação espontânea e sua influência em variáveis intracranianas merece investigações complementares.

Måis evidente foi a redução da PPC, claramente determinada pelo opióide, a partir de M2. Neste sentido, parece haver influência nos valores de pressão arterial, uma vez que tanto a PAS, quanto a PAM e PAD distaram significativamente a partir de $M 2$, dos valores colhidos em M1. Como a pressão de perfusão cerebral é obtida de relação matemática, subtraindo-se da PAM o valor da PIC, e uma vez que a pressão intracraniana permaneceu praticamente estável, durante 0 período experimental, pode-se observar que a redução da PPC foi determinada pelo efeito da buprenorfina sobre a hemodinâmica sistêmica, pela redução da pressão arterial. Este achado permite cotejar a assertiva de Stepien et al. (1995), segundo a qual o opióide causa alterações mínimas na pressão arterial; com os resultados atuais, não pode ser descartada a interação entre a buprenorfina e o sevoflurano, o que favoreceria a redução da pressão e seus reflexos na PPC.

Finalmente, cabe lembrar que a redução da PPC não pode ser, a princípio, considerado um efeito negativo da administração do opióide, sendo até benéfico, por exemplo, no caso de trauma cranioencefálico. Neste tipo de situação, a anestesia com a associação em teste pode ser viável.

Quanto à freqüência e débito cardíacos, parece claro o efeito da buprenorfina, fármaco que determinou a redução dos valores de modo significativo; desta forma foi possivel corroborar os achados de Stepien et al. (1995). Esta situação não deve ser revestida de preocupações, quando a anestesia for destinada a pacientes classificados como ASA I ou II, mas deve ser avaliada com maiores cuidados em animais em condições clínicas mais graves e pode ser totalmente contraindicada numa grande quantidade de casos, notadamente os que envolvem hipovolemia. A mesma assertiva é válida quando se observam os efeitos da buprenorfina sobre a PAP, com o agravante do possível reflexo desta variável sobre a oxigenação e shunt pulmonar.

Não foi possível cotejar os achados deste experimento com aqueles citados por: Bernard et al. (1990) e Kawana et al. (1995), que encontraram aumento da FC, com o sevoflurano, uma vez que o momento inicial de mensuração previa os pacientes já sob efeito do anestésico e os demais tempos de observação incluiam a interferência da buprenorfina, sobre o parâmetro. Entretanto, pelos valores encontrados em M1 tanto de FC como das demais variáveis hemodinâmicas, é possível suspeitar que se encontra revestida de verdade a afirmativa de Ebert et al: (1995), que trata da estabilidade cardiovascular propiciada pelo sevoflurano.

Sobre a temperatura corpórea, a redução observada pode ser imputada principalmente ao agente volátil, uma vez que sua redução foi constante e não houve aumento na taxa de decréscimo, após a aplicação da buprenorfina. $O$ efeito pode ser explicado, tanto pela redução observada do metabolismo basal, como descrito por Massone (1999), quanto da redução da pressão arterial. Esta última, por ser determinada também por vasodilatação periférica, permitiria troca de calor entre o sangue e o meio ambiente com maior eficiência (Nunes et al., 1995), levando, conseqüentemente, à diminuição do parâmetro.

Devido à pequena diferença observada entre os valores de TIC e os de TC, não é possível inferir a qualquer dos fármacos em teste influência direta sobre a temperatura intracraniana, sendo que a variação do parâmetro encontrada provém, provavelmente, do efeito dos fármacos sobre a temperatura corpórea, como já discutido.

\section{Conclusões}

Com base nos resultados obtidos, com a metodologia empregada, pode-se concluir que o opióide buprenorfina não interferiu na pressão intracraniana. Entretanto, a queda evidente da pressão arterial, como conseqüência da administração do fármaco, determina redução da pressão de perfusão cerebral em cães anestesiados com sevoflurano. Outrossim, os demais parâmetros cardiovasculares não sofreram influência do opióide, demonstrando estabilidade ao longo da anestesia geral inalatória.

\section{Agradecimento}

Os autores agradecem à Fundação de Amparo à Pesquisa no Estado de São Paulo (FAPESP) pelo apoio financeiro concedido.

\section{Referências}

BAGLEY, R.S.; KEEGAN, R.D., GREENE, S.A., HARRINGTON, M.L., MOORE, M.P. Pathologic effects in brain after intracranial pressure monitoring in clinically normal dogs, using a fiberoptic monitoring system. Am. J. Vet. Res., v. 56, n. 11, p. 1475-1478, 1995.

BERNARD, J.M., WOUTERS, P. F., DOURSOUT, M. F., FLORENCE, B., CHELLY, J. E., MERIN, R. G. Effects of sevoflurane and isoflurane on cardiac and coronary dynamics in chronically instrumented dogs. Anesthesiology, v. 72, n. 4, p. 659-662, 1990.
EBERT, T.J., HARKIN, C.P., MUZI, M. Cardiovascular responses to sevoflurane; a review. Anesth. Analg., v. 81, n. 6, p. 11-22, 1995.

GÓRNIAK, S. L. Hipnoanalgésicos e neuroleptoanalgesia. In: SPINOSA, H. S.; BERNARDI, M. M.; GÓRNIAK, S. L. (Ed.). Farmacologia aplicada à medicina veterinária. Rio de Janeiro: Guanabara Koogan, 1997, p. 140-146.

GREENE, S.A., HARTSFIELD S.M., TYNER, C.L. Cardiovascular effects of butorphanol in halothane-anesthetized dogs. Am. J. Vet. Res., V. 51, n. 8, p. $1276-1279,1990$. 
GROSS, M. E., SMITH, J.A., TRANQUILLI, W. J. Cardiorespiratory effects of combined midazolam and butorphanol in isoflurane-anesthetized cats. Vet. Surg., v. 22, n. 2, p. 159-162, 1993.

KAWANA, S., WACHI, J., NAKAYAMA, M., NAMIKI,A. Comparison of haemodynamic changes induced by sevoflurane and halothane in paediatric patients. Can. J. Anaesth., v. 42, p. 603-607, 1995.

MARTINEZ, E.A., HARTSFIELD, S. M., MELENDEZ, L. D., MATTHEWS, N.S., SLATER, M.R. Cardiovascular effects of buprenorphine in anesthetized dogs. Am. J. Vet. Res., v. 58, n. 11, p. 1280-1283, 1997.

MASSONE, F. Anestesiologia veterinária. Rio de Janeiro: Guanabara Koogan, 1999. 252 p.

NAVARRO, R., WEISKOPF, R. B., MOORE, M.A., LOCKHART, S., EGER, E. I., KOBLIN, D., LU, G., WILSON, C. Humans anesthetized with sevoflurane or isoflurane have similar arrhythmic response to epinephrine. Anesthesiology, v. 80, p. 545-549, 1994.
NUNES, N., POMPERMAYER, L.G; PIROLO, J.; RAHAL, S.C. Emprego do metaraminol no bloqueio da hipotensão produzida pela levomepromazina em cães. Braz. J. Vet. Res. An. Sci., v. 32, n. 2, p. 120-124, 1995.

OMOIGUI, S. Manual de drogas usadas em anestesia. 2. ed. São Paulo: Livraria.Santos Editora, 1998. 566 p.

SCHRAMM, W.M., STRASSER, K., BARTUNEK, A., GILLY, H., SPISS, C.K. Effects of rocuronium and vecuronium on intracranial pressure, mean arterial pressure and heart rate in neurosurgical patients. $\mathrm{Br}$. J. Anaesth., v. 77, n. 5, p. 607-611, 1996.

STEPIEN, R. L., BONAGURA, J. D., BEDNARSKI, R. M., MUIR III, W.W. Cardiorespiratory effects of acepromazine maleate and buprenorphine hydrochloride in clinically normal dogs. Am. J. Vet. Res., v. 56, n. 1, p. 78-84, 1995.

THURMON, J.C., TRANQUILLI, W.J., BENSON, G.J. Injectable anesthetics. In: Lumb \& Jones' veterinary anesthesia. 3. ed. Philadelphia: Lea \& Feabiger, 1996. p. 232-233. 